УAK 616.61-089.819.843-089.191.2-036.8

https://doi.org: 10.20538/1682-0363-2019-2-287-289

\title{
Endotypes of allergic rhinitis and asthma accompanying food allergy
}

\author{
Klimov A.V., Isaev P.Yu., Klimov V.V., Sviridova V.S. \\ Siberian State Medical University (SSMU) \\ 2, Moscow Tract, Tomsk, 634050, Russian Federation
}

\begin{abstract}
61 people suffering from persistent allergic rhinitis and asthma accompanying food allergies were studied using case histories, the NHANES questionnaire, polyspecific serum levels, allergen-specific IgE, IL4, IFNg and IL10 assays, and allergy skin tests. Four different endotypes have been identified, including entopic, which can be the basis for new approaches to the diagnosis and treatment of allergic rhinitis and asthma.
\end{abstract}

Key words: allergic rhinitis, asthma, food allergy, endotypes, entopy, allergens, IgE, cytokines, allergy skin tests.

Conflict of interest. The authors declare that there were no conflicts of interest related to this publication. Sourse of financing. The authors declare that there was no funding associated with this publication.

For citation: Klimov A.V., Isaev P.Yu., Klimov V.V., Sviridova V.S. Endotypes of allergic rhinitis and asthma accompanying food allergy. Bulletin of Siberian Medicine. 2019; 18 (2): 287-289. https://doi.org: $10.20538 / 1682-0363-2019-2-287-289$.

Persistent allergic rhinitis and bronchial asthma are the two main clinical manifestations of chronic atopy of the respiratory tract. The upper and lower airways cannot be separated from each other and can be treated with different immunomodulatory approaches, such as allergen-specific immunotherapy, as well as drugs that affect both compartments of the respiratory tract. Type II inflammation due to the Th2-controlled response to allergens in rhinitis and asthma is common, although these atopic conditions are characterized by some heterogeneity [1].

There are three aspects of heterogeneity: phenotype, endotype and biomarkers.

Phenotype is a classification category, which is determined by genotype, hereditary epigenetic factors and environmental factors. An endotype is a classification that is defined by various cellular and molecular pathways that underlie functional or pathobiological mechanisms. Endotypes include such features as clinical and immunological characteris-

Klimov Andrew V., e-mail: klimov.lor@mail.ru. tics, histopathology, upper and lower physiology, and the response to therapy for allergic rhinitis and asthma. Biological markers are specific parameters attributed to both phenotypes and endotypes [2-4].

A cohort of atopic patients of both sexes was studied in order to identify different endotypes and differentiate different therapeutic approaches associated with antigen-specific therapy and topical glucocorticoids. All patients $(n=61)$, aged 18 to 60 years, suffered from persistent allergic rhinitis that was accompanied by mild to moderate asthma, and 21 of them had food allergies to the most common allergens in adults, such as cow's milk, shrimp, peanuts, chicken eggs and wheat flour. Twenty healthy volunteers of the same age served as a control group.

A study of the case, family, social and occupational histories, questionnaire NHANES [5], physical and functional airway examination, serum polyspecific and allergenspecific IgE to Dermatophagoides pteronissinus (Der p 1), common major food allergens (nBos d 4, rPen a 1, nAra h 2, nGal d 2, and rTri a 19), IL4, IFNg, IL10, and allergic skin tests were carried out. 
The "cluster" analysis enabled dividing the patients into four groups, or endotypes (see Table).

T a ble

\begin{tabular}{|c|c|c|}
\hline \multicolumn{3}{|c|}{ Endotypes of atopic conditions } \\
\hline Entity & Endotype & $n$ \\
\hline 1 & $\begin{array}{l}\text { Classical atopic endotype with } \\
\text { subclinical food sensitization }\end{array}$ & 15 \\
\hline 2 & $\begin{array}{c}\text { Classical atopic endotype with food } \\
\text { allergy }\end{array}$ & 21 \\
\hline 3 & $\begin{array}{c}\text { Classical atopic endotype with no food } \\
\text { allergy }\end{array}$ & 10 \\
\hline 4 & Entopic endotype [6] & 15 \\
\hline
\end{tabular}

The correlation analysis for the whole cohort demonstrated the close correlation $(r=0.88$, $p<0.002)$ between serum IgE to Dermatophagoides pteronissinus (Der p1) and concentration of IL4 that confirmed atopic nature of all aspects of the investigated cases (see Fig.).

It was established that the classical atopic endotypes were in total characterized by high serum concentration of polyspecific IgE, general Th2 polarization, the frequency of positive allergy skin tests, and atopic family heredity. The classical atopic endotype with subclinical food sensitization was differentiated by the high level of oral tolerance due to many described mechanisms [7].

Interestingly, the food sensitization in patients of this endotype was the same as in patients of the other classical atopic endotype with clinical signs of food allergy.

The absence of sensitization to food proteins and, correspondingly, food allergy symptoms may be linked to the high, well-controlled, oral tolerance [8].

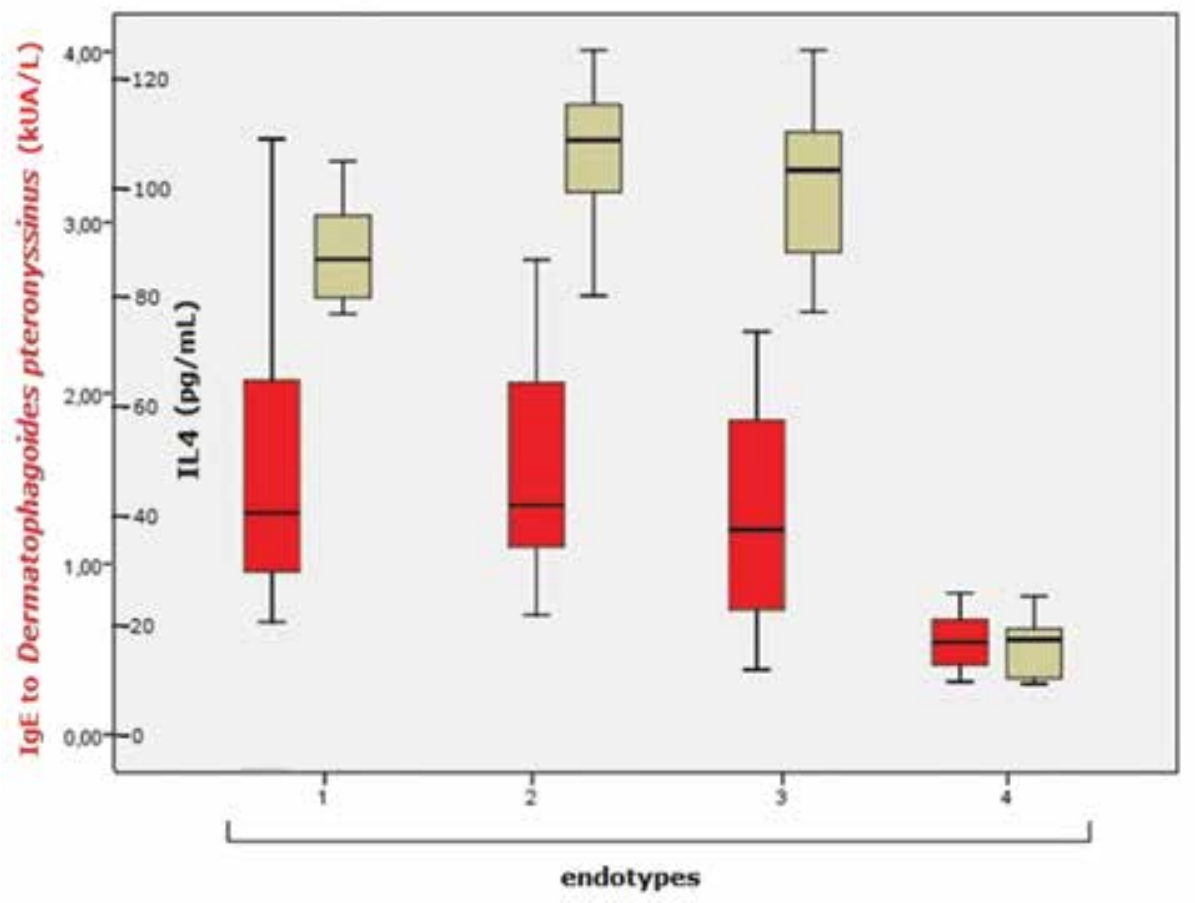

Figure. Correlation between serum IgE to dust mites and IL4

Entopy or entopic endotype is a new phenomenon discovered in allergology and immunology several years ago [6]. As our investigations showed, almost all parameters were the same as in healthy persons, and there are no systemic allergy signs. Nowadays, clinicians such as ENT specialists and lung physicians are involved in a discussion related to the diagnosis, treatment, and the relationship between local allergy and conventional or systemic allergy. Currently, the term "local rhinitis" is widely used, whereas there are only two references to "local asthma" $[9,10]$. However, a positive response to omalizumab in "non-allergic" severe asthma was described $[11,12]$ that demonstrated the presence of atopic IgE-dependent inflammation in such patients.

Atopic conditions are characterized by heterogeneity and may accompany the covert or clinical food sensitization, which enables downregulating the course of any atopic disease. The identification of atopic endotypes will promote and drive innovative developments in both allergen-specific immunotherapy and anti-inflammatory approaches, including severe asthma. 


\section{REFERENCES}

1. Bonilla F.A., Oettgen H.C. Adaptive immunity. J. Allergy Clin. Immunol. 2010; 125 (2): 33-40. DOI: 10.1016/j. jaci.2009.09.017.

2. Baker M.G., Sampson H.A. Phenotypes and endotypes of food allergy: path a to better understanding the pathogenesis and prognosis of food allergy. Ann. Allergy, Asthma and Immunology. 2018; 120 (3): 245-253. DOI: 10.1016/j.anai.2018.01.027.

3. De Greve G., Hellings P.W., Fokkens W.J. et al. Endotype-driven treatment in chronic upper airway diseases. Clinical and Translational Allergy. 2017; 7 (22): 1-14. DOI: 10.1186/s13601-017-0157-8.

4. Froidure A., Mouthuy J., Durham S.R. Asthma phenotypes and IgE responses. Eur. Respir. J. 2015; 12: 1-16. DOI: 10.1183/13993003.01824-2014.

5. Liu A.H., Jaramillo R., Sicherer S.H. et al. National prevalence and risk factors for food allergy and relationship to asthma: results from the National health and nutrition examination survey 2005-2006. J. Allergy Clin. Immunol. 2010; 126: 798-806. DOI: 10.1016/j. jaci.2010.07.026.

6. Powe D.G., Bonnin A.J., Jones N.S. 'Entopy': local aller- gy paradigm. Clin. Exp. Allergy. 2010: 40 (7): 987-997. DOI: $10.1111 / \mathrm{j} .1365-2222.2010 .03536 . x$.

7. Berin M.C., Shreffler W.G. Mechanisms underlying induction of tolerance to foods. Immunol. Allergy Clin. North Am. 2016; 36: 87-102. DOI: 10.1016/j.iac.2015.08.002.

8. Bryce P.J. Balancing tolerance or allergy to food proteins. Trends Immunol. 2016; 37 (10): 659-667. DOI: 10.1016/j. it.2016.08.008.

9. Kılıç E., Ali Kutlu A., Hastalıkları G. et. al. Does local allergy (entopy) exists in asthma? J. of Clinical and Analytical Medicine. 2016. Letters to Editors from 01.02.2016. DOI: $10.4328 /$ JCAM.3272.

10. Klimov V.V. From basic to clinical immunology. Springer Nature Switzerland AG 2019: 377. DOI: 10.1007/9783-030-03323-1.

11. De Llano L.P., Vennera M.C., Alvarez F.J. et al. Effects of omalizumab in non-atopic asthma: results from a Spanish multicenter registry. J. Astbma. 2013; 50 (3): 296-301. DOI: $10.3109 / 02770903.2012 .757780$.

12. Garcia G., Magnan A., Chiron R. et al. A proof-ofconcept, randomized, controlled trial of omalizumab in patients with severe, difficult-to-control, nonatopic asthma. Chest. 2013; 144 (2): 411-419. DOI: 10.1378/ chest.12-1961.

\section{Authors information}

Klimov Andrew V., PhD, MD, Assistant Professor, ENT Unit, SSMU, Tomsk, Russian Federation.

Isaev Pavel Yu., MD, Assistant Professor, Immunology and Allergy Unit, SSMU, Tomsk, Russian Federation.

Klimov Vladimir V., PhD, ScD, MD, Professor, Immunology and Allergy Unit, SSMU, Tomsk, Russian Federation.

Sviridova Valentina S., PhD, MD, Associate Professor, Immunology and Allergy Unit, SSMU, Tomsk, Russian Federation.

(凹) Klimov Andrew V., e-mail: klimov.lor@mail.ru.

Received 19.02.2019

Accepted 10.04.2019 